Quaderni di Geografia Cahiers de Gécongaty rataphie

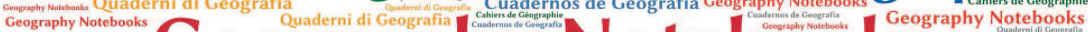
Cuadernos de Geografia Geography Notebooks $U 201000$ Cahiers de Géographi Cahiers de Géographie cahiers de Géographie Cuadernos de Geografía Cahiers de Géographie Cahiers de Géographie Cuadernos de Geografía Geography Notebooks

\author{
$4(2021)$ \\ 1 \\ Teatro di suoni. \\ Spazi acustici teatrali e territoriali
}

A cura di

Martino Mocchi, Lorena Rocca, Demis Quadri and Carlotta Sillano

EDITORIAL

Teatro di suoni per l'attaccamento ai luoghi. Uno sguardo geografico 11

Lorena Rocca

Per un teatro di suoni. Riflessioni su possibili dimensioni sonore nelle 23 creazioni site-specific di physical theatre

Demis Quadri

INTRODUCTION

Teatro di suoni. Spazi acustici teatrali e territoriali

Demis Quadri e Lorena Rocca

SPECIAL Issue

I suoni di Mantova come strumenti di interpretazione del paesaggio.

Tra turismo sostenibile ed educazione al patrimonio culturale

Valeria Pecorelli, Franca Zuccoli, Alessandra De Nicola, Enrico Squarcina

Il paesaggio sonoro campano tra contemporaneità e nuove forme

di progettualità turistica

Germana Citarella 
La narrazione spettacolarizzata del paesaggio sonoro.

Da Giuseppe Chiari a Philip K. Dick e oltre

Francesco Michi

Musica di paesaggi sonori. Enunciazione, risignificazione, comunicazione

Carlotta Sillano

Camminare per ascoltare. Partiture invisibili del territorio abitato

Elisabetta Senesi

Il paesaggio sonoro in relazione. Suono, movimento e immagini per stimolare complessità percettiva Angela Calia

Groove Fields. Understanding the Dance Floor from an Art-Based Research Perspective

Sebastian Mattbias

Il silenzio come esperienza trasformativa. L'importanza del silenzio nella meditazione e in ambito professionale

Sebastiano Caroni

Progettare il silenzio. Una lettura acustica dell'ex villaggio sanatoriale 125 Morelli a Sondalo

Martino Mocchi

Voicing One's Will. Theatre as Audio-Visual Hypotyposis of the Poetic

Michael Groneberg

Music and Clowning in Europe, 20th-21st centuries

Anna Stoll Knecht

Il paesaggio sonoro nella composizione musicale. Un percorso bibliografico

Stefano Alessandretti

\#exploreART: il labirinto di A. Pomodoro e i bambini. Un progetto di fruizione condivisa con percorsi sensoriali partecipati Alessandra De Nicola, Franca Zuccoli 


\section{OTHER EXPLORATIONS}

Il rumore lontano. Intervista a Lorena Rocca

a cura di Martino Mocchi

Re Cervo. Intervista a Antonella Astolfi

a cura di Krizia Bonaudo e Demis Quadri

Centovalli-Centoricordi. Intervista a Oliviero Giovannoni a cura di Krizia Bonaudo e Demis Quadri

Alla ricerca di un metodo: Open Space Technology 



\title{
Il paesaggio sonoro campano tra contemporaneità e nuove forme di progettualità turistica
}

\author{
Germana Citarella
}

Dipartimento di Scienze Politiche e della Comunicazione, Università degli Studi di Salerno

DOI: https://doi.org/10.7358/gn-2021-001-cita

\begin{abstract}
Full awareness of landscape occurs when it is holistically perceived. In other words, when there is osmosis between subject and object in terms of our observing, touching, breathing and listening to the same. Contemporaneously, the diminished attention paid to information provided by acoustic perception has had the effect of generating a propensity for looking rather than listening. The contribution intends to illustrate the soundscape of Campania through the tammurriata. It is not just one of the many forms of popular music expression, but the dance contains the focal points of a complex experience of collective participation, resulting in a significant tool for territorial knowledge, capable of promoting alternative forms of tourism and sense of community.
\end{abstract}

Keywords: soundscape; folk music; tammurriata; community based tourism; local development.

Parole chiave: paesaggio sonoro; musica popolare; tammurriata; turismo di comunità; sviluppo locale.

\section{INTRODUZIONE}

Nella cultura contemporanea predomina una forma di conoscenza che avviene attraverso lo sguardo e il senso della vista e questo particolare aspetto risulta essere maggiormente enfatizzato nell'attuale era digitale. Secondo alcuni studiosi che hanno indagato le forme di oralità nelle 
civiltà del passato (Havelock 1973, 2005; Ong 1986) il predominio della conoscenza visiva è subentrato allorquando la scrittura si è imposta come modalità principale di trasmissione della cultura già nell'antica Grecia ai tempi di Platone e - ancor di più - durante il Rinascimento quando appare, per la prima volta, la stampa. Tuttavia, grazie anche al crescente interesse suscitato dal processo percettivo, è emersa la convinzione che nella comprensione dei luoghi intervengano in maniera significativa gli altri sensi che, cogliendo la varietà dei suoni, dei sapori, degli odori, rivelano gli elementi caratteristici di un paesaggio. Eppure, la minore attenzione alle informazioni fornite dalla percezione acustica ha generato una propensione a guardare piuttosto che ad ascoltare, trascurando le atmosfere uditive dei luoghi (Minidio 2005). Invece, la percezione sonora del paesaggio è quella che si impone maggiormente poiché consente di cogliere la dimensione temporale di un luogo, in quanto il suono ha una durata ed una intensità variabile nel tempo (Cortesi 2010). Smith (2009), infatti, sosteneva che conoscere il mondo attraverso il suono è fondamentalmente diverso dal conoscerlo attraverso la vista, per questo, nel tempo, è cresciuta la necessità di guardare il paesaggio attraverso le sue sonorità ${ }^{1}$ per esaltare una nuova prospettiva della relazione tra lo spazio e l'uomo. In questo senso, la dimensione sonora, come strumento di investigazione dei luoghi, è stata presa in considerazione da varie discipline scientifiche tra cui quelle geografiche che, negli ultimi anni, hanno cercato di osservare il territorio non solo per le sue caratteristiche fisiche, economiche e sociali o per la struttura insediativa e l'organizzazione spaziale - pur importanti per la delineazione di un quadro territoriale - ma anche per il suo patrimonio di carattere culturale e per le componenti materiali, immateriali e semantiche che lo contraddistinguono (Cortesi 2010).

Su tali premesse si fonda il presente contributo, che intende illustrare il paesaggio sonoro della Campania attraverso una delle sue forme espressive più antiche e genuine che contribuisce, tutt'oggi, a plasmare la cultura sonora di una comunità, determinandone il suo localismo: la tammurriata. Quest'ultima - tramandata nei tempi antichi con il nome di canzuna 'e copp' 'o tammurro - non è semplicemente una forma di espressione musicale popolare ma racchiude i punti focali di una complessa esperienza di partecipazione collettiva, organizzati in una sequenza

${ }^{1}$ Infatti, la norma UNI ISO 12913-1: 2015 definisce il paesaggio sonoro come un "ambiente sonoro così come percepito, vissuto e compreso da una o più persone in uno specifico contesto". 
narrativa di suoni che permettono intuitivamente di percepire quello che accade oltre il dato visibile. In quest'ottica, la tammurriata - insieme a un vasto repertorio di canti ed espressioni musicali popolari quali la quadriglia, il ballo carnevalesco del laccio d'amore, il bello zampito e la "ndrezzata ischitana - può rappresentare non solo un importante strumento cognitivo per la conoscenza delle specificità territoriali storiche e culturali, ma anche una preziosa fonte di informazione, capace di concorrere al processo di percezione collettiva delle realtà locali. Essa, in tal modo, si trasforma in uno "strumento endogeno di autorappresentazione identitaria" (Giorda and Imarisio 2003, 59) capace di promuovere forme alternative di turismo come quello di comunità.

\section{Due PASSI INDIETRO NEL TEMPO: LA TAMMURRIATA}

In un saggio fondamentale intitolato Soundscape, comparso sulla rivista "Area" dell'Institute of British Geographers nel 1994, Susan Smith si propone di indagare il concetto di paesaggio sonoro nell'ambito degli studi di geografia sociale. Partendo dal concetto di 'paesaggio come testo' - contenuto nei lavori di Denis Cosgrove e Steve Daniels (Cosgrove 1984; Cosgrove and Daniels 1988) - la studiosa critica il limite imposto dalle consuetudini nell'accostarsi al paesaggio con un approccio pressoché visivo e silenzioso così da mettere in ombra gli altri sensi e, in particolare, il suono che ha il potere di evocare un 'senso del luogo' diverso da quello richiamato dalla vista. Infatti, gli uomini non possono vivere senza dare un senso a ciò che li circonda: di conseguenza, lo spazio da essi occupato è eterotopico (Foucault 1967), in quanto costituito da elementi improntati a significati tanto fisici che metafisici. Dunque, il paesaggio, così interpretato, segna il punto d'incontro tra il noto e l'ignoto e la ricerca del suo potenziale espressivo sarà realmente completata solo attribuendo valore anche alle testimonianze musicali che esprimono le tensioni emotive ed affettive tanto dell'individuo quanto della collettività a cui questi appartiene (Lucchesi 2012; Scaramellini 2007). In tal senso, la musica, intesa come 'racconto' identitario dei luoghi e come 'narrazione' del profondo legame che ogni comunità instaura con il proprio territorio, consente di ripercorrere e comprendere la storia della società, ricordando avvenimenti straordinari, ma soprattutto facendo rivivere luoghi significativi, i quali rappresentano punti fermi che trasmettono sensazioni eterne ed immutabili, nonostante il fluire del tempo. 
In tale contesto, un ruolo fondamentale è svolto dalle tradizioni musicali popolari ricche di informazioni che hanno varcato i secoli con la sola risorsa della voce e che l'etnomusicologo Diego Carpitella definisce come «'insieme dei sistemi di vita legati alle forme più arcaiche di economia: l'agricoltura, la pastorizia e la pesca» $(1988,57)$. Un genere tipico della tradizione orale è la tammurriata o ball'ncopp 'o tammurro ${ }^{2}$ caratteristica del mondo contadino della Campania ed in particolare delle pendici del Vesuvio e dei Monti Lattari.

Il Vesuvio si erge su un'ampia base circolare e si specchia nelle acque del Golfo di Napoli con la sua mole conica, recinta in parte dalla cerchia semidemolita del Monte Somma. Le pendici del vulcano risultano fittamente terrazzate: i muri a secco, costruiti con le scoriacee rocce ignee, sono molto spessi in modo da impedire la discesa del terreno e, nello stesso tempo, trattenere le cospicue quantità di ceneri e di terra fine che le piogge temporalesche trascinano dall'alto del monte, formando limo prezioso per una realtà agraria caratterizzata da un'elevata complessità e varietà colturale.

Invece, sull'orizzonte marino di Napoli «si innalza la barriera dei Monti Lattari, che sembra continuare nell'isola di Capri e che, illuminata dal sole pomeridiano, rivela la sua complessa morfologia» (Ruocco 1976, 523). Essi sono una catena montuosa, in parte dolomitica, interessata da numerose fratture trasversali, con una parete a picco sul Golfo di Salerno e con l'altra degradante dolcemente verso quello di Napoli. Dai Monti Lattari scendono impetuosi torrenti che un tempo alimentavano cartiere e attualmente consentono l'irrigazione degli agrumeti, che si estendono nei fondivalle o si arrampicano sulle falde terrazzate dei monti.

Ogni terrazzo era collegato attraverso un intricato sistema di canali di irrigazione alimentati da ruscelli, sorgenti e cisterne di

${ }^{2}$ Il canto è definito 'sul tamburo' poiché il corpo del cantatore è sempre proteso verso il suonatore, al fine di orientare la voce verso l'interno dello strumento per utilizzarne la cassa acustica. È uno stile relativamente omogeneo, nel quale si innestano espressioni regionali o locali, che presentano varietà di linguaggi musicali, di sfumature dialettali e di movimenti che caratterizzano il ballo legati anche alle abilità dei singoli esecutori. Attualmente si distinguono quattro tipologie diverse di ball'ncopp 'o tammurro: 1) quello domiziano caratterizzato da una forte corrispondenza tra il repertorio sonoro e musicale; 2) quello vesuviano prevede forme coreutiche ben riconoscibili; 3) quello lattaro presenta un ritmo a terzine sostenuto dalla vibrazione persistente della pelle del tamburo; 4) quello sarnese-nocerino contempla una maggiore pantomimica e una riduzione degli elementi strutturali tipici della tammurriata. 
captazione delle piogge. Su questo sistema si organizzano i centri urbani con le abitazioni che hanno come basamento i terrazzamenti, le scalette di accesso costruite seguendo le linee d'acqua così come le stradine per questo chiamate lavinare, le terrazze dotate di un giardino pensile formate dal terrapieno superiore. Le abitazioni seguendo la trama di scorrimento delle acque e i diritti di accesso agricolo si svolgono a spirali successive dalla costa alla montagna, seguono l'andamento dei terrazzi coltivati, sono esse stesse terrazze e giardini. Risultano assemblate l'una sull'altra e la terrazza giardino è sempre una parte integrante della casa stessa, rivestendo una funzione essenziale per la vita quotidiana: è luogo di lavoro, di comunicazione, di aggregazione. (Laureano 2004, 84-97)

Le sintetiche peculiarità sin qui descritte, non solo identificano e contraddistinguono il territorio Vesuviano e quello dei Monti Lattari, ma diventano anche fattori di ispirazione artistica che si ritrovano come ambientazione e sfondo nelle tradizionali tammurriate che, connesse al vissuto popolare, rappresentano lo spazio scenico entro il quale il cantore, accompagnato dal suono della tammorra ${ }^{3}$, impiega le proprie doti vocali e mimiche ${ }^{4}$. Pertanto, rappresenta l'occasione per esprimere le angosce, le delusioni e le paure quotidiane: è un metodo efficace per esorcizzare

${ }^{3}$ La tammorra, tammurro o tamburo è lo strumento musicale principale: scandisce il ritmo e il suono è prodotto dalla vibrazione della membrana di pelle tesa su un telaio circolare di faggio, legno compatto e resistente ma estremamente flessibile. Nella cornice vengono realizzate delle nicchie rettangolari, al cui interno sono inseriti dei sonagli detti 'e cicere o 'e cimbale ricavati da vecchie scatole di latta. Altri elementi di supporto all'esecuzione della tammurriata sono: 1) le castagnette, impugnate dai ballatori, costruite con legno di limone o di acacia selvatica del Vesuvio e seguono il ritmo del tamburo per tutto il tempo della danza; 2) il triccaballacche, strutturato da tre martelli di legno molto resistente: uno centrale fisso e gli altri mobili, tutti ancorati a due aste parallele. Si suona percuotendo ritmicamente i martelli laterali mobili contro quello centrale; 3) il putipù, un tamburo a frizione composto da una pentola di terracotta o da una scatola di latta tonda ricoperta da pelle di capra o di coniglio, nella cui parte centrale è stata applicata una canna e il cui fregamento produce delle vibrazioni amplificate dalla sottostante pentola o scatola che funge da cassa armonica.

${ }^{4}$ Ancora oggi il territorio vesuviano funge da palcoscenico naturale per l'ambientazione della tammurriata come, ad esempio, a Somma Vesuviana ( $\mathrm{Na}$ ) dove il sabato dopo Pasqua la comunità locale sale sul ciglio del Monte Somma per poi scendere a ritmo delle tammorre sino al sagrato del Santuario di Santa Maria a Castello. Inoltre, nello stesso Comune, la mattina del 3 maggio, i fedeli si riuniscono in un luogo denominato 'o Vallo$n e$, nei pressi dello stesso Santuario, dove ballano a suon di tammorra fino a tarda notte. 
le cause del disagio sociale attraverso un'azione catartica «che veicola una vera e propria maieutica del corpo sociale collettivo» (Barbuti 2007, 2). Gli avvenimenti, le storie di vita personali e familiari, passando di bocca in bocca, si traducono in una forma sintatticamente organizzata e funzionale per la memorizzazione, fino a diventare - attraverso uno sforzo di trasmissione generazionale - parte del bagaglio della tradizione comunicativa popolare.

Le origini della tammurriata risalgono al V secolo a.C. quando gli antichi greci giunsero in Italia, creando delle colonie in tutto il Meridione. I canti della popolazione ellenica eseguiti con il tamburo si diffusero nell'entroterra campano, anch'esso fortemente ancorato al mondo agricolo e al culto delle divinità che preservavano la semina e il raccolto. Il periodo della vendemmia, della mietitura, della raccolta delle olive, i momenti del ciclo produttivo della vita rurale erano indissolubilmente legati a occasioni gioiose: i coltivatori, come rito propiziatorio, attraverso il movimento dei loro corpi, assecondavano il ritmo regolare e simmetrico della tammorra ed esaltavano la fertilità della terra, unico strumento per il loro sostentamento.

Con l'avvento del Cattolicesimo la tammurriata si inserì nelle manifestazioni sacre e nei riti religiosi in onore della Madonna, considerata «vergine, madre, sorella, sposa, terra, ma anche come barca, mare in cui perdersi, caverna dalla quale si è nati e alla quale si vorrebbe sempre ritornare» (De Simone 1979, 13). Ancora oggi, le ricorrenze, le feste, i pellegrinaggi devozionali sono il più alto veicolo culturale aggregante che consente di recuperare la propria identità sociale e di partecipare collettivamente alla celebrazione del culto sacro con allegria e ilarità, suonando prima sul sagrato delle chiese e poi nei cortili delle abitazioni, dove un tempo il contadino curava la stanchezza a suon di tammorra e bevendo del vino ${ }^{5}$.

${ }^{5}$ Si riportano, di seguito, solo alcune delle tante occasioni in cui, ancora oggi, la tammurriata prende vita: 1) la sera di ogni 17 gennaio a Sant'Antonio Abate ed a Cicciano - in provincia di Napoli - si svolge la festa dei Fuochi di Sant'Antonio Abate durante la quale si rinnova il rito purificatorio e propiziatorio del falò in onore del Santo. Intorno ai fuochi accessi per le strade e nei cortili, la gente sosta fino a tarda notte, mangiando, bevendo e ballando a ritmo di tammurriata; 2) la mattina di ogni lunedì in Albis a Sant'Anastasia (Na), si svolge la festa dei Fujenti caratterizzata dall'arrivo continuo, festoso, ma anche sofferto dei devoti in cerca di grazia che strisciando, percorrono la navata centrale del Santuario della Madonna dell'Arco sino ad arrivare al quadro che la raffigura. Mentre nella Chiesa si svolge il singolare rito sacro, all'esterno si esegue il rito profano della tammurriata; 3) invece, ogni lunedi dopo la Pentecoste, fin dalle prime luci 
Il canto sul tamburo è solitamente preceduto da testi eseguiti senza accompagnamento strumentale che un tempo gli agricoltori intonavano per comunicare durante il duro lavoro nei campi e che attualmente hanno la funzione di introdurre la danza e di accogliere coloro che decidono di prenderne parte. Il movimento è costituito da una gestualità ritualizzata che richiama azioni svolte quotidianamente dai braccianti come setacciare la farina, spezzare la pasta e che, nel momento collettivo, assumono un significato simbolico e magico. La struttura coreutica del ballo è rappresentata da un cerchio di persone che nasce spontaneamente non appena si inizia a suonare e a cantare e al cui interno si muove una coppia di ballerini (ballatori) che eseguono simultaneamente le medesime rappresentazioni senza preordinare alcuna coreografia. Il cerchio diventa il confine all'interno del quale l'energia prende forma, attraverso un'efficace comunicazione basata sul linguaggio del corpo, ed esprime la volontà umana di sfuggire alla linearità del tempo canonico o almeno di sospenderlo così da dimenticare ed esorcizzare le difficoltà della vita quotidiana. L'apice della danza è simboleggiato dalla votata, unica circostanza che corrisponde a un preciso ritmo del tamburo e che consente ai ballatori di ruotare velocemente, mentre gli astanti, commentando con grida di compiacimento, di approvazione, di incitamento, sottolineano l'importanza dell'evento. Durante l'esecuzione non esistono attori e spettatori, non vi sono ostacoli tra i partecipanti alla festa, né sussistono palcoscenici, ma tutti i presenti condividono la celebrazione del rituale che terminerà solo quando lo scambio energetico tra le persone cesserà.

Sulla base di quanto sin qui esposto, la tammurriata potrebbe rappresentare un importante vettore per lo sviluppo di un'economia virtuosa, ovvero la chiave di volta su cui costruire un nuovo modello di organiz-

del mattino, folti gruppi di fedeli si dirigono sulla montagna che sovrasta Maiori (Sa). La meta della devozione è costituita da una grotta in cui è posto un altare e da una Chiesa, entrambe dedicate alla Madonna che dal 1485 è detta Avvocata. Sul monte le paranze mangiano, cantano e ballano in onore della Vergine e al tramonto scendono verso Maiori al suono della tammorra. Il livello di partecipazione a questa festa è altissimo; il percorso è vago, non prestabilito: tutto si svolge spontaneamente. Nulla può disturbare il rituale, mancano persino le bancarelle di dolciumi. L'unica merce che si vende è rappresentata dai limoni, frutto ritenuto magico dall'antica credenza, secondo la quale, basta mordere un limone per essere eternamente legato a chi lo ha offerto; 4) infine, il 16 ottobre di ogni anno a Materdomini di Caposele (Av) si svolge il culto in onore di San Gerardo Maiella. Esso si irradia nelle aree geografiche più distanti, raggiungendo, infatti, la Basilicata, il Lazio e la Puglia. Proprio la presenza di fedeli provenienti dalle zone più disparate favorisce una commistione di canti e balli che spaziano dalla tammurriata alla tarantella del Salento. 
zazione territoriale in grado di conciliare l'esigenza di tutela ambientale con quella di sviluppo socio-economico fondato sulla valorizzazione delle tradizioni culturali popolari attraverso forme di turismo alternative capaci di indagare le funzioni simboliche e metaforiche di un paesaggio per comprenderne gli aspetti eminentemente culturali, «i suoi depositi mitici e memoriali, le sue funzioni narrative» (Gambino 1997, 11).

\section{LA TAMMURRIATA COME STRUMENTO DI PROMOZIONE TURISTICA DEL TERRITORIO CAMPANO}

Sempre più frequentemente la scelta di una destinazione e il motivo di un viaggio sono legati a un'esperienza, a un evento a cui assistere, a un'occasione unica da vivere attraverso i sensi. La musica - intesa come proposta e pretesto di viaggio capace di coniugare un avvenimento culturale unico e irripetibile ai luoghi che diventano palcoscenico naturale dell'esecuzione stessa - si rivela un tramite comunicativo straordinario e universale in grado di muovere un numero significativo di persone che concorrono a creare un indotto nei luoghi in cui si realizzano le manifestazioni.

Nonostante la Campania sia un contesto territoriale vivace e dotato di attrattori di qualità - tanto da aver registrato 6.075 milioni di arrivi e 21.132 milioni di presenze turistiche nel solo anno 2018 (BMT 2019) - la grande possibilità che la tammurriata offre a quelle realtà dove le popolazioni locali - in assenza di un effettivo riconoscimento sociale non possono rivendicare alcun empowerment è quella di partecipare alla ricostruzione sociale (Martinotti 1993) del proprio territorio per affrontare le sfide del presente e del futuro tra cui la ricerca di forme alternative di turismo. Queste ultime, al di là degli obiettivi imprescindibili della sostenibilità ambientale e della competitività territoriale, da un lato devono prevedere la costruzione di un sistema integrato di offerta che esprima, tanto negli orientamenti attrattivi quanto nelle soluzioni ricettive, una forte connotazione identitaria dall'altro, la creazione di meccanismi di integrazione dell'offerta che guidino la domanda a una fruizione turistica consapevole, capace cioè di cogliere le specificità del contesto territoriale e di tradursi effettivamente in quell'esperienza dell'alterità che costituisce il fondamento motivazionale del viaggio.

Le priorità descritte permetterebbero alla comunità di: 1) acquisire una maggiore conoscenza della propria realtà geografica, al fine di migliorare la gestione delle risorse; 2) controllare l'offerta del prodotto 
turistico, affinché rispetti la capacità di carico dei luoghi; 3) garantire una destinazione più competitiva in grado di offrire un prodotto completo, coerente e adeguato alle esigenze di specifici target di domanda ma saldamente ancorato alla dimensione identitaria del luogo. Ciò consentirebbe non solo all'ospite di intessere relazioni profonde con i luoghi e con le persone, permettendogli di accrescere il proprio background di esperienze e di stimolare nuove suggestioni, ma garantirebbe anche consistenti ricadute economiche su tutta la filiera del comparto turistico, migliorando la qualità della vita della popolazione locale. Quanto sostenuto trova probabilmente la sua più compiuta espressione nel turismo di comunità, che più delle altre forme è in grado di esprimere e rappresentare l'identità territoriale. In particolare, adattandosi facilmente alla struttura sociale del territorio, è in grado di integrarsi senza traumi con la realtà umana, sociale e culturale della destinazione, contribuendo positivamente a un sano recupero delle attività, delle consuetudini, delle peculiarità e dei valori ambientali del sito. Esso, infatti, offre l'occasione di non cadere nel circolo vizioso del prodotto turistico di massa in quanto la comunità non è più deputata a essere solo sede organizzativa delle attività ricettive, ma si pone come soggetto attivo in grado di guidare il turista alla scoperta del territorio campano mediante un'esperienza musicale emotivamente coinvolgente e di abituarlo a reputare la tipicità un valore aggiunto. Lo sviluppo di questa importante nicchia di mercato costituisce, pertanto, una preziosa risorsa per evitare la globalizzazione e la standardizzazione dell'offerta, attraverso la costruzione e l'accrescimento delle capacità locali, nel quadro di un modello di sviluppo in cui la compatibilità ambientale, sociale ed economica sia considerata criterio guida per un rapporto corretto e produttivo con i luoghi. Naturalmente, la rilevanza strategica di questo particolare segmento dell'offerta turistica dipende dal suo livello di territorializzazione, ossia dall'essere effettivamente espressione delle forze locali capaci di preservare le valenze culturali del territorio e promuovere forme di fruizione compatibili con le peculiarità del contesto. In assenza di queste caratteristiche, il community based tourism non solo perde le valenze appena menzionate, ma può anche involvere verso forme di banalizzazione o anche di mistificazione della realtà territoriale in cui si dipana. 


\section{ConClusioni}

La musica - benché sia un bene immateriale - al pari dei suoni, delle luci e degli odori non solo concorre alla descrizione del paesaggio geografico al punto da caratterizzarlo e da lasciare un'impronta indelebile nell'immaginario collettivo ma, in quanto esternalità positiva fruibile localmente, genera un movimento di persone motivate dal desiderio di ascoltarla.

Nello specifico, la tammurriata - che affonda le sue radici nell'antica civiltà ellenica - pur potendo divenire un fattore di crescita economica e di competitività territoriale, a mio avviso non deve essere letta e interpretata unicamente come proposta turistica, ma anche come momento di riappropriazione sociale, simbolica e fisica del territorio, capace di migliorare l'efficienza relazionale della comunità.

In altri termini la comunità locale, attraverso la tammurriata, riprende coscienza di sé, si rende conto del valore del proprio patrimonio e si impegna a tutelarlo, rinvigorirlo e alimentarlo perché padrona della sua lettura e interpretazione.

Di conseguenza, compito delle istituzioni dovrebbe essere quello di sostenere l'ascesa del turismo di comunità per promuovere iniziative e manifestazioni tese a tramandare di generazione in generazione le tradizioni musicali popolari, come la tammurriata, consentendo così ad ambiti territoriali che occupano una posizione geografica marginale di poter trovare una giusta collocazione nel sistema turistico locale.

\section{RIFERIMENTI BIBLIOGRAFICI}

Alberti, C. 2017. Tammurriata. Riti e miti di una sirena millenaria. Napoli: Valtrend Editore.

Amedeo, G. 2005. Canzoni e popolo a Napoli dal ‘400 al '900. Napoli: Grimaldi Editore.

Barbuti, M. 2007. La tammurriata. Mito, fede e tradizione nella cultura contadina della Campania (Italia).

Borsa Mediterranea del Turismo. 2019. Il posizionamento della Campania nella prospettiva del Rapporto sul Turismo italiano. Convegno 23 marzo 2019, Napoli.

Carpitella, D. 1988. "Lingue e cultura popolare". In Lingue e culture locali. Lingua e cultura nazionale una disciplina multidisciplinare, a cura di E. Zuanelli Sonino, 56-59. Padova: Cleup Editore.

Cortesi, G. 2010. “Un'ipotesi di valorizzazione culturale del territorio”. Geografia e organizzazione dello sviluppo territoriale. Studi regionali e monografici 52: 7-11. 
Cosgrove, D. 1984. Social formation and Symbolic Landscape. London: Croom Helm.

Cosgrove, D. and S. Daniels, eds. 1988. The iconography of landscape: essays on the symbolic representation, design and use of past environments. Cambridge: Cambridge University Press.

De Simone, R. 1979. Canti e tradizioni popolari in Campania. Roma: Lato Side Editori.

Foucault, M. 1967. Dits et écrits. Des espaces autres. Conference au Cercle d'études architecturales, 14 marzo 1967.

Gambino, R. 1997. Conservare, innovare. Paesaggio, ambiente, territorio. Torino: Utet.

Giorda, C. e S. Imarisio. 2003. "Processi di sviluppo territoriale nelle Valli di Lanzo: milieu, rete locale dei soggetti, rappresentazione del territorio, identità del patrimonio culturale". In Tracce di SloT in provincia di Torino: il caso di studio valli di Lanzo, a cura di Cristiano Giorda, 39-80. Dipartimento Interateneo Territorio.

Havelock, E. A. 1973. Cultura orale e civiltà della scrittura. Bari: Laterza [trad. it. Mario Carpitella].

Havelock, E. A. 2005. La musa impara a scrivere. Riflessioni sull'oralità e l'alfabetismo dall'antichità al giorno d'oggi. Bari: Laterza [trad. it. Mario Carpitella].

Laureano, Pietro. 2004. "I terrazzamenti del territorio campano". In La coltura dei terrazzamenti per la salvaguardia del paesaggio, a cura di Pietro Laureano, 84-97. Salerno: Edizioni Menabò.

Lucchesi, F. 2012. "Sviluppi teorici e tematiche di indagine negli studi di geografia umanistica: i paesaggi letterari e quelli cinematografici”. Annali della Facoltà di Lettere e Filosofia dell'Università degli Studi di Milano 65 (2): 193-220.

http://www.ledonline.it/acme/.

Martinotti, G. 1993. Metropoli. La nuova morfologia sociale della città. Bologna: Il Mulino.

Mauro, G. 2006. Gli strumenti musicali tradizionali della Campania. Napoli: Simeoli Editore.

Minidio, A. 2005. I suoni del mondo. Studi geografici sul paesaggio sonoro. Milano: Guerini.

Ong, W. 1986. Oralità e scrittura. Bologna: Il Mulino [trad. it. Alessandra Calanchi].

Ruocco, D. 1976. Campania. Torino: Utet.

Scaramellini, G. 2007. "Luoghi e culture, la "dimensione geografica". In Tradizioni e modernità. Saperi che ci appartengono, a cura di Giorgio Botta, 79-144. Torino: Giappichelli.

Smith, B. R. 2009. "Sintonizzarsi su Londra intorno al 1600”. In Paesaggi sonori, edited by Michael Bull and Les Back, 87-95. Milano: Il Saggiatore [trad. it. Franco Fabbri e Alessandra Gallone].

Smith, S. J. 1994. “Soundscape”. Area 26 (3): 232-240. http://www.jstor.org/ stable/20003453.

Vicinanza, P. 2005. Tammurriata anima e corpo. Sorrento: Franco Di Mauro Editore. 